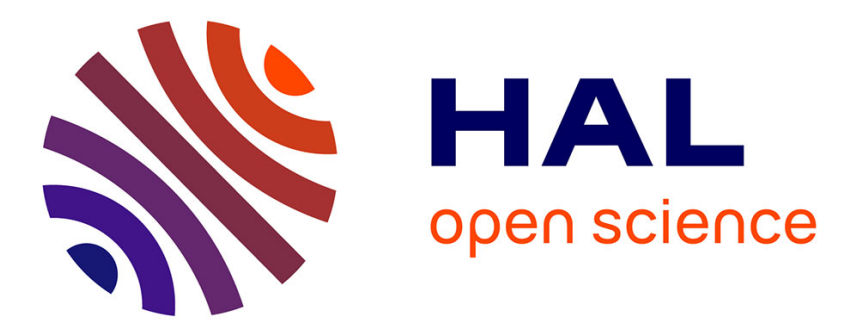

\title{
Post-treatment positron emission tomography-computed tomography is highly predictive of outcome in Plasmablastic lymphoma
}

\author{
Yassine Al Tabaa, Emmanuelle Tchernonog, Pierre Faurie, Anne Ségolène \\ Cottereau, Hélène Monjanel, Antoine Bonnet, Steven Le Gouill, Emmanuel \\ Gyan, Hervé Ghesquières, Denis Mariano-Goulart, et al.
}

\section{To cite this version:}

Yassine Al Tabaa, Emmanuelle Tchernonog, Pierre Faurie, Anne Ségolène Cottereau, Hélène Monjanel, et al.. Post-treatment positron emission tomography-computed tomography is highly predictive of outcome in Plasmablastic lymphoma. European Journal of Nuclear Medicine and Molecular Imaging, 2018, 45 (10), pp.1705-1709. 10.1007/s00259-018-4020-5 . hal-02340143

\section{HAL Id: hal-02340143 \\ https://hal.science/hal-02340143}

Submitted on 7 Jan 2020

HAL is a multi-disciplinary open access archive for the deposit and dissemination of scientific research documents, whether they are published or not. The documents may come from teaching and research institutions in France or abroad, or from public or private research centers.
L'archive ouverte pluridisciplinaire HAL, est destinée au dépôt et à la diffusion de documents scientifiques de niveau recherche, publiés ou non, émanant des établissements d'enseignement et de recherche français ou étrangers, des laboratoires publics ou privés. 


\title{
Post-treatment positron emission tomography-computed tomography is highly predictive of outcome in Plasmablastic lymphoma
}

\author{
Yassine Al Tabaa ${ }^{1} \cdot$ Emmanuelle Tchernonog $^{2} \cdot$ Pierre Faurie $^{3} \cdot$ Anne Ségolène Cottereau $^{4} \cdot$ Hélène Monjanel $^{5}$. \\ Antoine Bonnet $^{6}$. Steven Le Gouill ${ }^{6}$. Emmanuel Gyan ${ }^{5} \cdot$ Hervé Ghesquieres $^{3}$ - Denis Mariano-Goulart ${ }^{1}$. \\ Guillaume Cartron ${ }^{2}$
}

\begin{abstract}
Purpose Plasmablastic lymphoma (PBL) is a rare variant of diffuse large B cell lymphomas (DLBCL) clinically characterized by a poorer prognostic. Few clinical and imaging data are available and derived from pooled case reports and small series. The aim of the study was to evaluate the FDG avidity at baseline and the utility of 18-Fluorodeoxyglucose (FDG) positron-emissiontomography/computed-tomography (PET/CT) for staging and response assessment.

Methods Patients with newly diagnosed PBL seen at Lymphoma Study Association centers during the period 2005-2015 were included if they underwent a PET/CT at staging and at the end of treatment (eotPET) and had received an anthracycline-based first line therapy. EotPET scans were analyzed using the 5-point-scale visual analysis in accordance with Lugano criteria. Patients were classified in complete metabolic response (CMR) or no-CMR including partial metabolic response (PMR), stable disease (SD) and progression disease (PD). EotPET results were assessed for the ability to predict event free survival (EFS) and overall survival (OS).

Results Thirty-five PBL patients fulfilled the inclusion criteria. The median follow-up was 34 months (2.8-120 months). FDG avidity was found in all patients at diagnosis. Most patients $(80 \%)$ achieved CMR, and 20\% were no-CMR including 9\% PMR, 6\% SD, and 6\% PD. A CMR after first line chemotherapy predicted higher EFS $(p<0.0001)$ and OS $(p=0.0006)$.
\end{abstract}

Conclusions This study confirmed the FDG avidity of PBL subtype and the usefulness of PET/CT scanning in restaging an aggressive lymphoma at the completion of chemotherapy. EotPET can predict outcomes following treatment in patients with PBL.

Keywords 18 -FDG-PET/CT $\cdot$ FDG avidity $\cdot$ Plasmablastic lymphoma $\cdot$ Post-treatment evaluation

\section{Introduction}

Yassine A1 Tabaa

yassine.altabaa@gmail.com

Department of Nuclear Medicine, CHU Montpellier, 80 Avenue Augustin Fliche, 34095 Montpellier, Cedex 05, France

Department of Hematology, University Hospital of Montpellier, UMR CNRS 5235, Montpellier, France

Léon Bérard Center, Department of Hematology, Lyon, France

Department of Nuclear Medicine, CHU Tenon, Paris, France

Department of Hematology, University Hospital of Tours,

Tours, France

Department of Hematology, University Hospital of Nantes, INSERM UMR 892, Nantes, France
Plasmablastic lymphoma (PBL) is a tumor entity initially described in 1997, and now considered as a subtype of diffuse large B cell lymphoma (DLBCL) $[1,2]$. This rare variant of DLBCL is typically associated with immunodeficiency state, especially HIV infection and post-transplant patients, but has also been described in immunocompetent patients, and accounts for approximately $2 \%$ of acquired immune deficiency syndrome related lymphoma [3]. Epstein-Barr virus (EBV) seems to be closely associated with the development of PBL especially in immunocompromised patients [4]. A clinically aggressive course and poor prognostic are usually reported, without consensus for the treatment, since most clinical data were derived from cases reports or small series. The role of immunosuppression reduction, local radiotherapy, intensified 
chemotherapy, anti-CD20 monoclonal antibody or autologous hematopoietic stem cells transplantation remains largely unknown.

Response evaluation is a key issue in defining the best treatment strategy for lymphoma patients, and 18Fluorodeoxyglucose positron emission tomography/ computed tomography (18-FDGPET/CT) recently emerged as an important tool for chemosensitivity evaluation and response assessment in aggressive 18FDG-avid lymphomas. Because PBL is not clearly reported yet to be an FDG-avid non-Hodgkin lymphoma subtype, 18-FDGPET/CT is not currently recommended in the modified international working group response criteria to stage, survey, and assess treatment response. Thus, data in PBL are still sparse and 18-FDGPET/ CT results have been only reported in few isolated case reports [5-7]. One such approach could be useful to identify PBL patients with inferior outcomes who may be candidates for novel approaches and research protocols. We have conducted a retrospective cohort study to examine 18 -FDG avidity and prognostic utility of 18-FDG-PET/CT imaging in PBL patients undergoing dose-intensive chemotherapy.

\section{Materials and methods}

\section{Study patients}

We retrospectively screened for eligible patients from the Lymphoma Study Association (LYSA) and newly diagnosed as PBL between June 2005 and June 2015 as part of a previously published study [8]. Inclusion criteria were diagnosis of PBL established by histopathology, immunohistochemistry examinations according to the WHO classification, PET/CT scans at diagnosis and for evaluation of treatment response (eotPET).

\section{8-FDG-PET/CT acquisitions}

Baseline PET/CT scans were performed within 4 weeks before initiation of first-line chemotherapy. EotPET were performed within 8 weeks of the completion of treatment. All patients were instructed to fast for at least $6 \mathrm{~h}$ before 18FDG injection. Serum glucose levels were measured using the hexokinase method. Whole-body emission and transmission scans were acquired in the 3D mode $60 \mathrm{~min}$ after intravenous administration of $3.5 \mathrm{MBq} / \mathrm{kg} 18-\mathrm{FDG}$. Nondiagnostic-quality non-contrast-enhanced $\mathrm{CT}$ images were acquired before acquisition of the PET data.

\section{Baseline and treatment response evaluation}

Quantitative assessment was performed for each metabolic target lesion using the standard uptake value (SUVmax). A
FDG avid lymphoma was defined as the presence of at least one focus of 18-FDG uptake reported at a disease site. A nonavid 18-FDG lymphoma was defined as disease proven by clinical examination, conventional imaging modalities, and histopathology, with no evidence of 18-FDG uptake in the involved sites.

Treatment response was assessed using the revised Lugano classification with the 5PS visual analysis with a cut-off value of grade 4 for positivity [9]. Patients were classified as having a complete-metabolic-response (CMR, 5PS score $<4)$, or non-complete-metabolic-response (no-CMR, 5PS score $\geq 4$ ) including partial-metabolic-remission (PMR), nonmetabolic-response (NMR) or progressive-disease (PD) according to results of the PET examination. SUV and delta SUV were not analyzed.

$\mathrm{CT}$ response was evaluated using the $\mathrm{CT}$ based response Cheson 2007 criteria [10].

\section{Study outcomes}

Event free survival (EFS) was defined as the time (in months) from PBL diagnosis to the time of documented disease progression or discontinuation of treatment for any reason. Overall survival (OS) was defined as the time (in months) from PBL diagnosis to death. Patients with documented disease progression or death or who were lost to follow-up were censored from further analysis.

\section{Statistical analysis}

We first described the survival experience for the study cohort using the Kaplan-Meier method. We then described the survival experience for subjects stratified on their PET/CT status at post-treatment. Survival curves were compared by the logrank test. All $P$ values of $<0.05$ were considered to indicate statistical significance. Multivariate analysis was performed using Cox regression methods. Statistical analysis was conducted using the R Core Team (R version 3.1.3, R-Foundation for Statistical Computing).

\section{Results}

\section{Characteristics of patients with PBL}

Of a total of 135 patients with newly diagnosed PBL [8], 19 patients did not have 18-FDG-PET/CT available at baseline, 28 patients underwent primary palliative care, and 53 patients with curative chemotherapy did not undergo PET/CT scans after treatment (Fig. 1). Characteristics of the 35 patients included in this analysis are presented in Table 1. The most commonly used regimen was CHOP or CHOP-like, in 27 cases. Six patients received anthracycline-based intensive 
Fig. 1 Flow chart of plasmablastic lymphoma (PBL) patients. PBL patients selected according to baseline and posttreatment 18-FDG-PET/CT evaluation

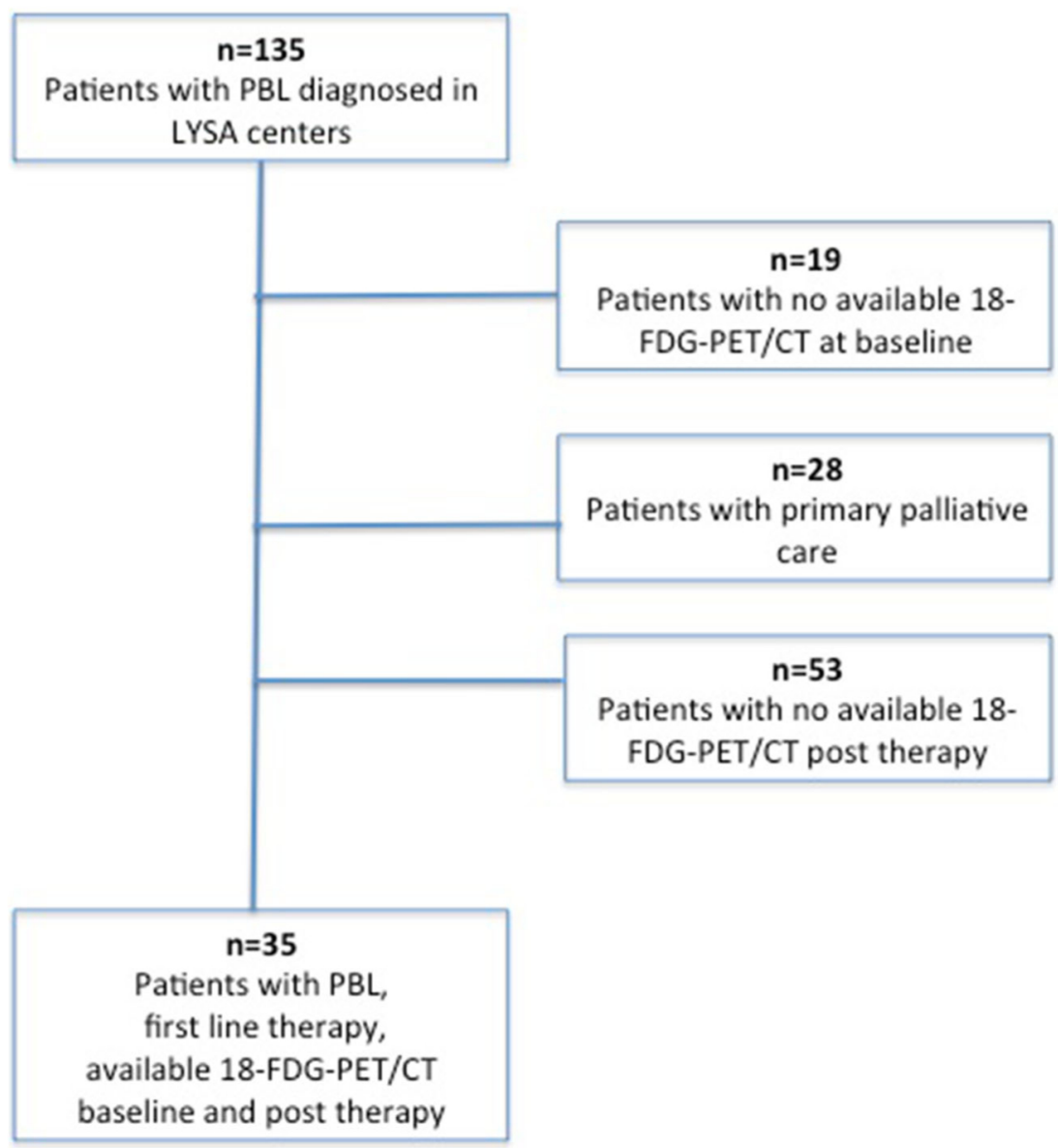

regimen ACVBP (doxorubicin, cyclophosphamide, vindesine, bleomycin, prednisone), EPOCH (etoposide, doxorubicin, vincristine, cyclophosphamide, prednisone), or
COPADEM (cyclophosphamide, vincristine, prednisone, adriamycin, methotrexate). Two patients received bortezomib and anthracyclin. Rituximab was given in 12 cases, either for
Table 1 Characteristics of study patients $(\mathrm{N}=35)$ and the initial cohort $(\mathrm{N}=135)$

\begin{tabular}{|c|c|c|c|c|c|}
\hline \multirow[t]{2}{*}{ Characteristic } & \multicolumn{2}{|l|}{ Total cases } & \multicolumn{2}{|c|}{ Cohort study } & \multirow[t]{2}{*}{ P-value } \\
\hline & $\mathrm{n}$ & $\%$ & $\mathrm{n}$ & $\%$ & \\
\hline Total & 135 & $(100 \%)$ & 35 & $(100 \%)$ & \\
\hline Male & 108 & $(80 \%)$ & 29 & $(83 \%)$ & NS \\
\hline Median age (years) (range) & $58(16-88)$ & & $57(16-85)$ & & NS \\
\hline VIH positive patients & 56 & $(42 \%)$ & 14 & $(40 \%)$ & NS \\
\hline Transplanted patients & 17 & $(12 \%)$ & 6 & $(17 \%)$ & NS \\
\hline \multicolumn{6}{|l|}{ Primary involved site } \\
\hline Oral cavity & 25 & $(19 \%)$ & 9 & $(26 \%)$ & NS \\
\hline Ear, nose, throat site & 20 & $(15 \%)$ & 6 & $(17 \%)$ & NS \\
\hline Gastro-intestinal tractus & 21 & $(16 \%)$ & 4 & $(11 \%)$ & NS \\
\hline \multicolumn{6}{|l|}{ Ann Arbor stage } \\
\hline I or II & $72 / 134$ & $(54 \%)$ & 17 & $(49 \%)$ & NS \\
\hline III or IV & $62 / 134$ & $(46 \%)$ & 18 & $(51 \%)$ & NS \\
\hline \multicolumn{6}{|l|}{ IPI score } \\
\hline $0-2$ & $87 / 125$ & $(70 \%)$ & $26 / 34$ & $(75 \%)$ & NS \\
\hline $3-5$ & $38 / 125$ & $(30 \%)$ & $8 / 34$ & $(23 \%)$ & NS \\
\hline \multicolumn{6}{|l|}{ Immunochemistry } \\
\hline CD20 & $13 / 127$ & $(10 \%)$ & $3 / 33$ & $(9 \%)$ & NS \\
\hline CD138 & $113 / 128$ & $(88 \%)$ & $32 / 35$ & $(91 \%)$ & NS \\
\hline EBER & $63 / 102$ & $(62 \%)$ & $20 / 30$ & $(67 \%)$ & NS \\
\hline Ki67 (\%median) & $80 \%$ & & $80 \%$ & & NS \\
\hline
\end{tabular}


CD20 weakly positive cases or to treat EBV reactivations. Four patients underwent autologous hematopoietic stem cell transplantation. Histopathological review was reviewed in $90 \%$ of cases.

The median follow-up was 34 months (range 2.8120 months), and no patient was lost to follow-up. At last follow-up, 9/35 patients (26\%) died. Death was related to lymphoma progression in eight cases and treatment-related toxicity in one case.

\section{8-FDG avidity in PBL}

Staging PET/CT scans were FDG-avid in all PBL patients (35/ 35 ), with a median SUVmax of 10 (range 4.1-40.2) at a suspected site of disease. Extranodal involvement was observed in $23 / 35$ patients $(66 \%)$ in the oral cavity, the ear nose throat sites, and the gastro intestinal tractus; $17 / 35$ patients had nodal involvement (49\%). Moreover, compared with CT, PET/CT upstaged 4 patients (staging Ann Arbor I to II in 2 patients, and III to IV in 2 patients).

\section{Metabolic response to treatment and survival analysis}

Twenty-eight patients (80\%) achieved CMR (ten patients with deauville 1 , ten with deauville 2 , and eight with deauville 3 ), and $7(20 \%)$ no-CMR including three patients (9\%) with PMR (deauville 4$)$, two patients (6\%) with SD (Deauville 4), and two patients (6\%) with PD (Deauville 5). Concerning conventional CT response, 29 patients $(83 \%)$ achieved CR, two patients $(6 \%)$ with PR, two patients $(6 \%)$ with $\mathrm{SD}$, and two patients $(6 \%)$ with PD. A difference between conventional $\mathrm{CT}$ and PET metabolic response was observed in one case (radiological complete response and partial metabolic response). Patients with a CMR had more localized stage and low IPI compared to patients who did not achieved CMR, even though it did not reach significance $(p=0.07$ and 0.08 , respectively). HIV infection, EBV viral load, EBER and cmyc expression were not correlated to PET response.

PBL patients with a positive post-treatment PET/CT were associated with a statistically significantly inferior EFS $(p<$ $0.0001)$ and $\mathrm{OS}(p=0.0006)$. The median EFS and OS survival based on post-treatment PET/CT status (positive vs negative) were 7 months vs 45 and 22 months vs 87 , respectively (Fig. 2). Among the clinical parameters, only IPI score was associated with EFS $(\mathrm{p}<0.0001)$ and $\operatorname{OS}(p=0.01)$.

\section{Discussion}

In our study, we confirmed that PBL is an FDG-avid nonHodgkin lymphoma subtype since all baseline PET/CT were positive. Moreover, post-therapy PET/CT scans are predictive of EFS and OS. Over the past decade, the utility of 18-FDG-

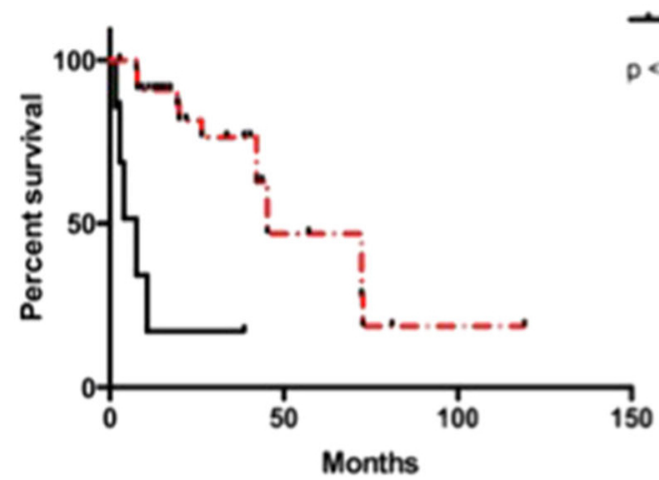

B

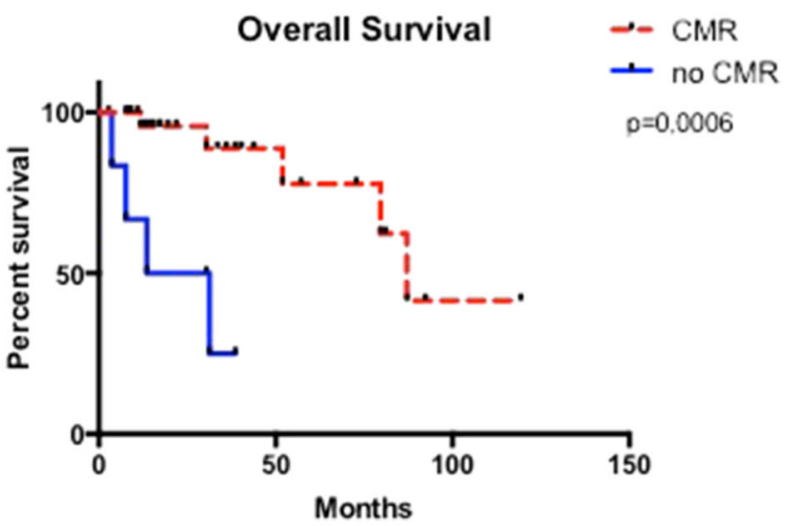

Fig. 2 Event free survival (EFS) and overall survival (OS) in plasmablastic lymphoma patients. EFS (A) and OS (B) according to treatment response using the revised Lugano criteria. Two groups of responders were defined by achieving a complete response (CMR group) or no-complete response (no-CMR group)

PET imaging in the management of lymphoma has markedly expanded. Whereas in DLBCL and Hodgkin lymphoma, there is a robust literature to support the use of PET/CT to identify patients at high risk for failure $[11,12]$, the data in other lymphoma subtypes are limited by the lack of prospective data, the heterogeneity of patient populations or treatment strategies, and most importantly, the lack of uniformity in the way FDG-PET imaging is interpreted. In the case of PBL, there was no evidence to support the use of FDG-PET for staging and response assessment since very few reports underlined the usefulness of PET/CT in PBL characterization and staging [5-7]. This recommendation is in part because of paucity of data available in the literature. Furthermore, no previous studies tested the ability of PET/CT to evaluate response to treatment in PBL. In our study, we used established objective criteria to define PET/CT status. Therefore, the patient population, treatment approach, and response criteria are clearly defined and hence reproducible for future prospective validation studies. This study is also the first attempt to test the use of PET/CT in restaging an aggressive lymphoma and evaluating response at the completion of chemotherapy using the Lugano criteria. In this small cohort, PET/CT upstaged 
$11 \%$ of patients compared with CT, and there was a good correlation between radiological and metabolic responses since difference between responses was observed in one case (radiological complete response but partial metabolic response; radiotherapy on the residual localization which permitted complete response).

Furthermore, we report that PBL is an FDG-avid NHL subtype. Indeed, this subtype is highly aggressive with a high Ki67 and a plasmablastic differentiation that could explain the FDG avidity. Therefore, pre-treatment 18-FDG-PET/CT could be recommended and should be incorporated into the design of future prospective clinical trials to validate these results.

Finally, our results demonstrate that $18-\mathrm{FDG}-\mathrm{PET} / \mathrm{CT}$ is highly predictive of outcome in PBL subtype in firstline therapy and appears to be useful to identify PBL patients with inferior outcomes who may be candidates for novel approaches and research protocols. However, the relative good survival in our cohort as compared to the initial cohort of 135 patients with a median OS and EFS, respectively, of 32 and 22 months [8] could be explained by (i) the selection of the patients having underwent baseline and eotPET, that is, intensive chemotherapy treated patients, excluding early death patients that corresponded to a selection bias, and (ii) the absence of recommendation for this rare entity. The other limitations of the study would be the retrospective analysis with a low percentage of patients undergoing both baseline and eotPET, due to the aggressive disease with many patients with palliative care with no available PET evaluation. Nevertheless, these limitations did not influence the good performances of PET/CT found in this entity.

In conclusion, this study suggests that 18 -FDGPET/CT is a promising imaging modality for diagnosis and response evaluation of PBL and could be recommended as well for all DLBCL subtypes. However, further prospective investigation with large patient enrolment is warranted to confirm these findings.

\section{Compliance with ethical standards}

Conflict of interest The authors declare that they have no conflict of interest.
Ethical approval All procedures performed in studies involving human participants were in accordance with the ethical standards of the institutional and/or national research committee and with the 1964 Helsinki declaration and its later amendments or comparable ethical standards. For this type of study formal consent is not required.

\section{References}

1. Delecluse HJ, Anagnostopoulos I, Dallenbach F, et al. Plasmablastic lymphomas of theoral cavity: a new entity associated with the human immunodeficiency virus infection. Blood. 1997;89(4):1413-20.

2. Stein H, Harris N, Campo E. Plasmablastic lymphoma. In: Swerdlow S, Campo E, Harris N, et al., editors. WHO classification of Tumours of the Haematopoietic and lymphoid tissues. 4th ed. IARC: Lyon; 2008. p. 256-7.

3. Carbone A. AIDS-related non-Hodgkin's lymphomas: from pathology and molecular pathogenesis to treatment. Hum Pathol. 2002;33(4):392-404.

4. Castillo JJ, Reagan JL. Plasmablastic lymphoma: a systematic review. Sci World J. 2011;11:687-96.

5. Makis W, Ciarallo A, Lisbona R. Plasmablastic lymphoma of the oral cavity in an HIV positive patient: staging with 18F-FDG PET/ CT. Acta Radiol. 2011;52(9):970-2.

6. Cazaentre T, Sanhes L, Laurent G, Costa K, Vallantin X, et al. Interesting image. Assessment of plasmablastic lymphoma by F-18 FDG PET/CT: case report. Clin Nucl Med. 2010;35(11):882-3.

7. Treglia G, Paone G, Stathis A, Ceriani L, Giovanella L. An unusual case of Plasmablastic lymphoma presenting as paravertebral mass evaluated by (18) F-FDG PET/CT. Nucl Med Mol Imaging. 2014;48(1):89-90.

8. Tchernonog E, Faurie P, Coppo P, et al. Clinical characteristics and prognostic factors of plasmablastic lymphoma patients: analysis of 135 patients from the Lysa group. Ann Oncol. 2016;28(4):843-848.

9. Cheson BD, et al. Recommendations for initial evaluation, staging, and response assessment of Hodgkin and non-Hodgkin lymphoma: the Lugano classification. J Clin Oncol. 2014;32(27):3059-68.

10. Cheson BD, Pfistner B, Juweid ME, et al. Revised response criteria for malignant lymphoma. J Clin Oncol. 2007;25:579-86.

11. Itti E, Meignan M, Berriolo-Riedinger A, et al. An international confirmatory study of the prognostic value of early PET/CT in diffuse large B-cell lymphoma: comparison between Deauville criteria and DeltaSUVmax. Eur J Nucl Med Mol Imaging. 2013;40:1312-20.

12. De Wit M, Bohuslavizki KH, Buchert R, Bumann D, Clausen M, Hossfeld DK. 18FDG-PET following treatment as valid predictor for disease-free survival in Hodgkin's lymphoma. Ann Oncol. 2001;12:29-37. 